\title{
Effect of a quinoa (Chenopodium quinoa $W$ ) based diet on the intestinal mucosa of growing Wistar rats
}

\author{
S. M. Vidueiros ${ }^{1}$, I. Fernandez ${ }^{1}$, D. Bertero ${ }^{2}$, M. E. Roux ${ }^{3}$ and A. Pallaro ${ }^{1}$ \\ ${ }^{1}$ Department of Nutrition, Faculty of Pharmacy and Biochemistry, University of Buenos Aires (UBA) and ${ }^{2}$ Department of \\ Vegetal Physiology, Faculty of Agronomy, UBA and ${ }^{3}$ ININCA, UBA-CONICET, Argentina
}

Quinoa (Chenopodium quínoa $W$ ) is a pseudocereal native to the Andean region of South America that has been recognized as an extremely nutritious grain all over the world ${ }^{(1)}$. In previous studies, we characterized the nutritional properties of quinoa from Argentinean Northwest ${ }^{(2,3)}$. Moreover, quinoa seeds have a high content of saponins that give them a bitter taste and may alter the intestinal mucosa due to their detergent properties ${ }^{(1)}$.

The aim of the present study was to find out the effect of a variety of quinoa from Campo Tapial de Colanzuli, Iruya, Salta, without washing treatment, on the intestinal mucosa of growing rats.

In this study, Wistar rats ( $n=6 /$ group) from weaning to 7 days fed a $10 \%$ protein diet with 1$)$ unwashed quinoa (Q), 2) commercial washed quinoa $(\mathrm{QR})$, and 3) casein (C) as control group. Body weight (BW, g) and diet intake (I, g/day) were determined, and Ponderal Growth Rate (PGR, g/day/100g) was calculated. Intestines were removed, processed by Saint Mariés technique and stained with Alcian Blue-H/E. Goblets cells/100 epithelial cells (GC) were determined in 10 intestinal villi/rat. Tissue sections were studied by indirect immunofluorescence technique $\left.{ }^{4}\right)$. IL- $17+$ cells, CD5 $+\mathrm{T}$ cells and TCR $\gamma \delta+$ subset levels were measured in lamina propria (LP) and intraepithelium (iIEL). IgA-B + cells were determined in LP by reading the number of cells/30 fields.

Results are shown in the table below.

\begin{tabular}{|c|c|c|c|c|c|c|c|}
\hline \multirow{2}{*}{ Group } & & \multicolumn{2}{|c|}{$\mathrm{Q}$} & \multicolumn{2}{|c|}{$\mathrm{QR}$} & \multicolumn{2}{|c|}{$\mathrm{C}$} \\
\hline & & Mean & SD & Mean & $\mathrm{SD}$ & Mean & SD \\
\hline BW & & $45.0^{\mathrm{a}}$ & 2.7 & $60.0^{\mathrm{b}}$ & 5.4 & $69.7^{\mathrm{c}}$ & $\overline{6.4}$ \\
\hline PGR & & $-1.97^{\mathrm{a}}$ & 0.6 & $1.63^{\mathrm{b}}$ & 0.5 & $3.95^{\mathrm{c}}$ & 0.9 \\
\hline I & & $4.7^{\mathrm{a}}$ & 0.3 & $6.9^{\mathrm{ab}}$ & 1.0 & $8.4^{\mathrm{b}}$ & 0.6 \\
\hline $\operatorname{IgA}-\mathrm{B}+$ & & 78.3 & 16.6 & 72.3 & 8.1 & 80.4 & 16.1 \\
\hline GC & & $24.2^{\mathrm{b}}$ & 6.5 & $14.7^{\mathrm{a}}$ & 2.7 & $10.9^{\mathrm{a}}$ & 1.2 \\
\hline \multirow[t]{2}{*}{ IL-17 + } & LP & $60.3^{\mathrm{b}}$ & 9.3 & $42.6^{\mathrm{a}}$ & 4.5 & $41.7^{\mathrm{a}}$ & 6.6 \\
\hline & iIEL & $12.0^{\mathrm{a}}$ & 4.7 & $9.6^{\mathrm{a}}$ & 2.9 & $10.0^{\mathrm{a}}$ & 2.4 \\
\hline \multirow[t]{2}{*}{$\operatorname{TCR} \gamma \delta+$} & LP & 71.8 & 5.4 & 66.3 & 6.9 & 58.7 & 13.3 \\
\hline & iIEL & 14.8 & 1.0 & 10.8 & 3.1 & 10.8 & 2.9 \\
\hline \multirow[t]{2}{*}{ CD5 + } & LP & 54.3 & 10.2 & 60.8 & 13.6 & 54.2 & 13.1 \\
\hline & iIEL & 9.8 & 1.5 & 9.8 & 2.9 & 9.3 & 5.5 \\
\hline
\end{tabular}

Means within rows with different superscript, differ $0.05>p<0.001$ (ANOVA, followed by SNK test).

No differences were observed in IgA-B + cells, CD5 + T cells and TCR $\gamma \delta+$ subset among groups. Moreover, goblets cells and IL-17+ cells were significant increased in Q group which could indicate an inflammatory process caused by the saponins from unwashed quinoa. This could affect the normal intestinal absorption of nutrients which is reflected in BW, PGR and I, compared to QR group.

This work was supported by UBACyT 20020100100255 and 20620100100014.

1. Izquierdo J, Mujica A, Jacobsen S, Marathee JP, (2001) FAO, Santiago (Chile). Oficina Regional para América Latina y el Caribe.

2. Vidueiros SM, Dyner L, Bertero D \& Pallaro A (2010) FASEB J 24, 949.2

3. Vidueiros SM, Peterson G, Bertero D \& Pallaro A (2011) FASEB J 25, 770.16.

4. Vidueiros SM, Fernandez I, Slobodianik N, Roux ME \& Pallaro A (2008). Nutrition 24, 575-581. 